\title{
Acceleration feedback-based active and semi-active seismic response control of rail-counterweight systems of elevators
}

\author{
Rildova and M.P. Singh* \\ Department of Engineering Science and Mechanics, Virginia Polytechnic Institute and State University, \\ Blacksburg, VA 24061, USA
}

Received 6 May 2005

Revised 28 June 2005

\begin{abstract}
Based on the observations in the past earthquake events, the traction elevators in buildings are known to be vulnerable to earthquake induced ground motions. Among several components of an elevator, the counterweight being heaviest is also known to be more susceptible than others. The inertial effects of the counterweight can overstress the guide rails on which it moves. Here we investigate to use the well-known acceleration feedback-based active and semi-active control methods to reduce stresses in the rails. The only way a control action can be applied to a moving counterweight-rail system is through a mass damper placed in the plane of the counterweight. For this, a part of the counterweight mass can be configured as a mass damper attached to a small actuator for an active scheme or to a magneto-rheological damper for a semi-active scheme. A comprehensive numerical study is conducted to evaluate the effectiveness of the proposed configuration of control system. It is observed that the two control schemes are effective in reducing the stress response by about 20 to $25 \%$ and improve the system fragility over a good range of seismic intensities.
\end{abstract}

Keywords: Elevators, earthquake motion, counterweight, dynamic response, system fragility, active control, semi-active control

\section{Introduction}

The people in a modern society depend heavily on the elevators for vertical transportation in almost all buildings of stories higher than three, and in all hospitals higher than one story. They serve a very important function in the buildings, but in essential facilities they are indispensable as one can not afford to have a malfunctioning elevator in a hospital dealing with an emergency arising after a strong earthquake. Past observations have shown that these systems are highly susceptible to the earthquake induced ground motions. Several design changes have evolved since the occurrence of San Fernando Earthquake in 1971, and they are now mandated by the ASME Elevator code [1]. These changes have certainly improved the performance of the elevator systems, but their malfunctions have still been reported in later earthquake events.

The elevators contain several electrical and mechanical components that can malfunction during an earthquake, but in a traction elevator the counterweight-rail system is considered to be the most vulnerable. It is primarily because the counterweight in an elevator is designed to balance the weight of the passenger car plus $40 \%$ of the total payload of the elevator. Thus being the heaviest component in an elevator, the counterweight provides large inertial forces during ground-induced vibrations. This mass combined with the flexibility in the system provided by the roller guide assemblies at each corner of the counterweight which guide the counterweight on a pair of rails with bracket

*Corresponding author. Tel.: +1 540231 4572; Fax: +1 540231 4574; E-mail: mpsingh @vt.edu. 
supports, the rail-counterweight system becomes a dynamical system. In an earthquake, the counterweight-rails receive multiple support motion filtered through the building, which causes it to vibrate both in the in-plane and out-of-plane directions. These dynamic effects have often damaged the counterweight rails and other hardware attached to the system. Several studies $[5,9,10,12]$ were conducted to examine the dynamic behavior of these systems with different models, including a few recent ones by the writers [4,6,7]. In the most recent study [7], the writers have used an analytical model of the counterweight with roller guides, including the flexibilities of all supporting and guiding components (e.g., roller guide assemblies, guide rails and support brackets). An important part of this model development has been the inclusion of gaps mandated by the ASME code [1] which introduce nonlinearities caused by closing and opening of these gaps as the motion proceeds. These code-mandated gaps are provided between the counterweight frame and the rails and also between the restraining plates and the rails to relieve the stresses in the rails and also to prevent excessive displacement and derailment of the counterweight during earthquake-induced vibrations. The dynamic analyses conducted with this model for several recorded and synthetic earthquake motion accelerograms have shown that commonly used size guide rails can get overstressed during normally expected ground motion intensities [7]. It is, therefore, desirable to investigate the protective methods that can help in reducing the vibration effects of the counterweights to improve the performance of elevator systems in earthquakes.

The stress in the guide rails caused by counterweight vibrations can be reduced by increasing the rail size and/or providing closer bracket supports if these options are possible. However, the maximum available rail size is the 30-lb rails and sometimes the option of reducing bracket spacing is not possible. The use of a tie bracket which connect the two rails is often considered, but our earlier study has shown that this only reduces the rail stress locally near the tie bracket with an almost equal increase at other locations [6,7]. Other options to reduce the dynamic effects are the use of passive devices like discrete dampers to enhance the system damping, or use active and semi-active devices that induce counteractive forces to reduce the system response. The tight spaces around a counterweight and the limited clearances between the counterweights and the rails limit the installation and full utilization of discrete dampers; our studies have shown that because of the limited clearances their effectiveness is not very significant. We have also examined the installation of a constrained viscoelastic layer at the bottom of the guide rails to enhance the damping in the systems, but it was found to be quite ineffective as it did not add damping between the main vibrating component (the counterweight) and the rails.

The other remaining option is to use active and semi-active systems, if arrangements can be made to apply forces to the counterweight. With the given layout of the system, the application of the forces in the out-of-plane direction on the counterweight is not feasible. It is, however, possible to apply forces in the in-plane direction, and the only feasible arrangement to this seems to be through an active mass driver or a mass damper. Herein, this approach is examined for the active and semi-active control of the in-plane vibrations. This approach does not reduce the out-of-plane vibrations, but in a counterweight rail system the in-plane vibrations are in any case more critical, sometime causing twice as high stresses in the rails as those caused by the out-of-plane motion.

Herein the arrangement shown in Fig. 1(b) is used to create a mass damper to apply the forces in the in-plane direction. Figure 1(a) shows the schematic of a counterweight along with the counterweight frame, rails, bracket supports, and a visible roller guide assembly. The counterweight consists of layers of dead weights stacked over each other in a counterweight frame. The whole counterweight-frame assembly is considered to act as a rigid body. Figure 1(a) shows the five dynamic degrees of freedom of this system: (a) translation $u$ and rotation $\psi$ describing the in-plane motion, and (b) translation $v$, and two rotations $\theta$ and $\phi$ describing the out-of-plane motion. The top layer of the dead weight can be reconfigured into a mass of a tuned mass damper which can be attached to a spring, damper and an actuator or a controllable semi-active device such as a MR damper braced against the counterweight frame. The tuned mass damper can roll on the stack of weights below on roller bearings. This arrangement is shown in part (b) of Fig. 1. In the following we study this system under active and semi-active modes and evaluate its effectiveness in reducing the stresses in the rails. We first describe the analytical models of the active and semi-active schemes used. No new control algorithms are developed; rather, the existing ones are used to generate numerical results to evaluate the effectiveness of the proposed control system. The effectiveness is measured both in terms of the stress reduction in the rails as well as in term of reducing the system fragility for seismic motions. 


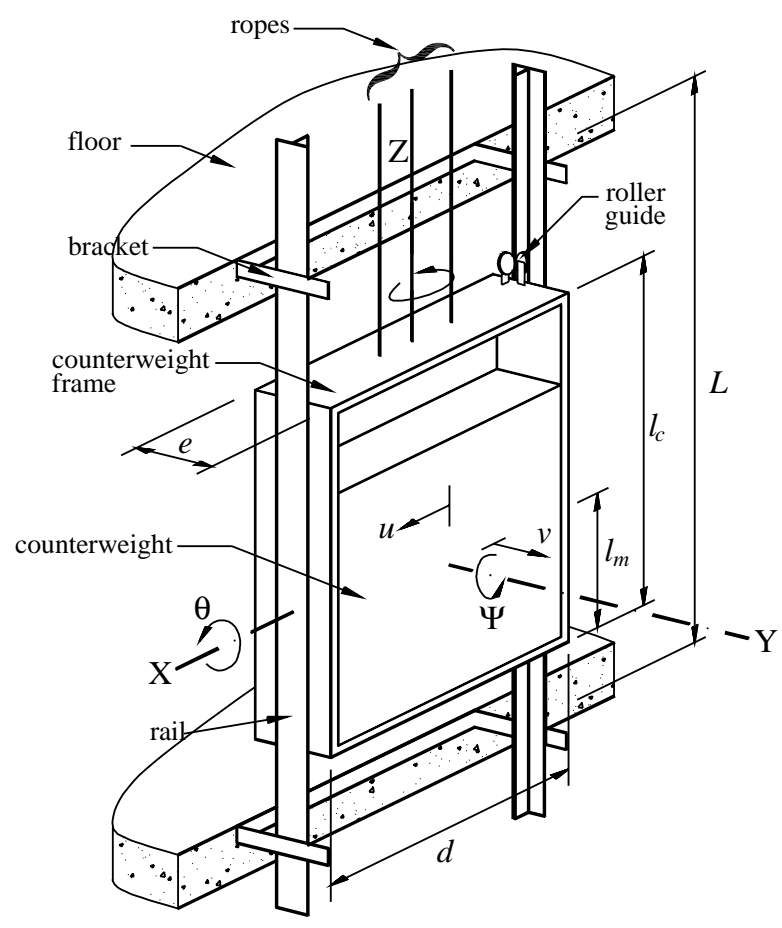

(a)

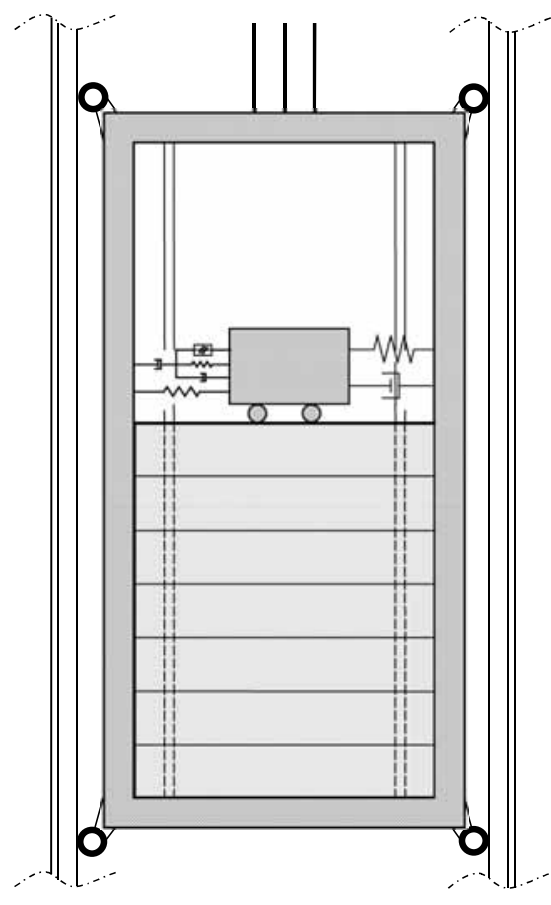

(b)

Fig. 1. (a) The sketch showing a counterweight and rails and five dynamic degrees-of-freedom, and (b) the proposed configuration of an in-plane tuned mass damper in the counterweight frame.

\section{Equations of motion}

The detailed development of the complete equations of motion is given in Reference [4]. Here we only provide the final set of equations primarily for the in-plane direction as the proposed active or semi-active devices are placed only in this direction. For comprehensive numerical evaluation of the effectiveness of the control systems, however, both the in-plane and out-of-plane motions have been considered. The in-plane equations of motion with in-plane control actions on the counterweight-rail system are written in the following standard form:

$$
\boldsymbol{M}_{i} \ddot{\boldsymbol{q}}_{i}+\boldsymbol{C}_{i} \dot{\boldsymbol{q}}_{i} \boldsymbol{K}_{i} \boldsymbol{q}_{i}=-\boldsymbol{M}_{i} \ddot{\boldsymbol{y}}_{i}+\boldsymbol{D} f_{T}+\boldsymbol{f}_{i}
$$

where $\boldsymbol{M}_{i}, \boldsymbol{C}_{i}$, and $\boldsymbol{K}_{i}$ are, respectively, the mass, damping, and stiffness matrices; $\boldsymbol{q}_{i}$ is the in-plane displacement vector; $\ddot{\boldsymbol{y}}_{i}$ is the acceleration input vector from the building floors; $f_{T}$ is the actuator force; $\boldsymbol{D}$ is the location vector; and $\boldsymbol{f}_{i}$ is the nonlinear force vector related to the contacts with the rails. The displacement vectors $\boldsymbol{q}_{i}$ is defined as:

$$
\boldsymbol{q}_{i}=\left\{\begin{array}{c}
u \\
u_{e} \\
u_{T}
\end{array}\right\}
$$

where $u$ is the in-plane relative displacement of the counterweight center of mass with respect to the building base; $u_{T}$ is the relative displacement of the TMD mass with respect to the base; and $u_{e}=l_{m} \psi$ is the displacement coordinate related to the in-plane rotational degrees of freedom in which $l_{m}$ is the distance from the bottom of the counterweight to its center of mass (Fig. 1a). The mass matrix is defined as:

$$
\boldsymbol{M}_{i}=m_{c}\left[\begin{array}{ccc}
1-\mu & 0 & 0 \\
0 & \frac{1}{3}\left(1-\frac{d^{2}}{4 l_{m}^{2}}\right) & 0 \\
0 & 0 & \mu
\end{array}\right]
$$




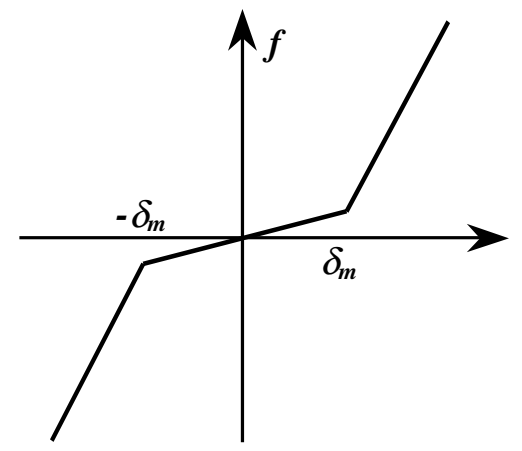

(a)

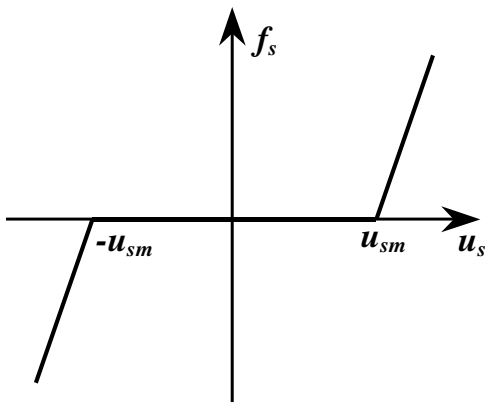

(b)

Fig. 2. Bilinear force-deformation diagram for the equivalent springs (a) at the roller guide assemblies, and (b) at the contact point of the counterweight frame with the rail.

where $\mu$ is the ratio of the mass damper to the total mass of the counterweight $m_{c}$ and dimension $d$ is shown in Fig. 1(a). The in-plane stiffness matrix is defined as:

$$
\boldsymbol{K}_{i}=\boldsymbol{T}_{i}^{\boldsymbol{T}}\left[\begin{array}{ccc}
k_{i u} & 0 & 0 \\
0 & k_{i l} & 0 \\
0 & 0 & k_{b r}
\end{array}\right] \boldsymbol{T}_{i}+k_{T}\left[\begin{array}{ccc}
1 & 1-\mu & -1 \\
1-\mu & (1-\mu)^{2} & -(1-\mu) \\
-1 & -(1-\mu) & 1
\end{array}\right]
$$

where the first term in this matrix represents the contributions of the counterweight support elements (roller guide assemblies, rails and bracket supports) and the second term represents the contribution of the spring element connecting the TMD mass with the frame. The coefficients $k_{i u}$ and $k_{i l}$ in Eq. (4) are the equivalent stiffness coefficients for the in-plane motion at the upper and lower corners of the counterweight, respectively, and $k_{b r}$ is the bracket stiffness coefficient. The equivalent stiffness coefficients include the flexibilities of the rails, bracket and helical springs in the roller guides, and are defined in References [4,6]. The equivalent springs have a bilinear force-deformation relationship, as shown in Fig. 2(a) where $\delta_{m}$ in the figure denotes the clearance at the restraining plate (3/16 in. in the code). The low stiffness part of the diagram represents the condition without contact, where the roller guide assembly governs the equivalent stiffness. The steep part of the diagram represents the contact condition, where only the rail and bracket supports contribute to the equivalent stiffness. Another bilinear force-deformation diagram, shown in Fig. 2(b), is added to represent contact between the frame and the rail when the counterweight is located on two consecutive span of the rail. In Fig. 2(b), $u_{s m}$ denotes the clearance between the frame and the rails (1/2 in. in the code). A more complete discussion of these nonlinearities and their effects on the seismic response of the rail-counterweight system is discussed in References [4,7]. The coefficient $k_{T}$ in Eq. (4) is the stiffness coefficients of the spring attached between the TMD and the frame. The presence of $\mu$ in Eq. (4) accounts for the eccentric placement of the damper spring in the frame. The transformation matrix $\boldsymbol{T}_{i}$ in Eq. (4) is defined as:

$$
\boldsymbol{T}_{i}=\left[\begin{array}{ccc}
1 & l_{c}-1 & 0 \\
1 & l_{m}-1 & 0 \\
1 & l_{c}-1-l_{s} & 0
\end{array}\right]
$$

The damping matrix is defined parallel to the stiffness matrix as follows:

$$
\boldsymbol{C}_{i}=\left[\begin{array}{ccc}
c_{11} & c_{12} & 0 \\
c_{21} & c_{22} & 0 \\
0 & 0 & 0
\end{array}\right]+c_{T}\left[\begin{array}{ccc}
1 & 1-\mu & -1 \\
1-\mu & (1-\mu)^{2} & -(1-\mu) \\
-1 & -(1-\mu) & 1
\end{array}\right]
$$

The first part of the damping stiffness is included to represent inevitable energy dissipation in the system, and the second part is due to the contribution of the damper attached between the TMD mass and the frame with damping coefficient $c_{T}$. The location vector $\boldsymbol{D}$ and the force vector $\boldsymbol{f}_{i}$ are defined as: 


$$
\boldsymbol{D}=\left\{\begin{array}{c}
-1 \\
\mu-1 \\
1
\end{array}\right\} ; \quad \boldsymbol{f}_{i}=\boldsymbol{T}_{i}^{\boldsymbol{T}}\left\{\begin{array}{ll}
u_{m} \Delta k_{i u} & \operatorname{sgn}\left(u_{u}\right) \\
u_{m} \Delta k_{i l} & \operatorname{sgn}\left(u_{l}\right) \\
u_{s m} k_{b r} & \operatorname{sgn}\left(u_{s}\right)
\end{array}\right\}
$$

where $\Delta k$ is the difference between the current equivalent stiffness and the lower value of stiffness when there is no contact.

The above equations are valid both for the active and semi-active control schemes. The only difference will be in the manner the force $f_{T}$ is determined. In both schemes it is convenient to work with the state form of the equation of motion:

$$
\dot{z}=\boldsymbol{A} \boldsymbol{z}+\boldsymbol{B} f_{T}+\boldsymbol{E} \ddot{\boldsymbol{y}}_{i}+\boldsymbol{f}^{*}
$$

where the state vector is defined as,

$$
z=\left\{\begin{array}{c}
\boldsymbol{q}_{i} \\
\dot{\boldsymbol{q}}_{i}
\end{array}\right\}
$$

and the state matrices as

$$
\boldsymbol{A}=\left[\begin{array}{cc}
0 & \boldsymbol{I} \\
-\boldsymbol{M}_{i}^{-1} \boldsymbol{K}_{i}-\boldsymbol{M}_{i}^{-1} \boldsymbol{C}_{i}
\end{array}\right] ; \quad \boldsymbol{B}=\left\{\begin{array}{c}
0 \\
\boldsymbol{M}_{i}^{-1} \boldsymbol{D}
\end{array}\right\} ; \quad \boldsymbol{f}^{*}=\left\{\begin{array}{c}
0 \\
\boldsymbol{M}_{i}^{-1} \boldsymbol{f}_{i}
\end{array}\right\} ; \quad \boldsymbol{E}=\left\{\begin{array}{c}
0 \\
-\boldsymbol{I}
\end{array}\right\}
$$

\section{Acceleration feedback control}

Herein the control force is determined using the well-established procedures of the linear optimal control in which a cost function of the following form is minimized.

$$
\boldsymbol{J}=\int_{0}^{\infty}\left(z^{T} \boldsymbol{Q} \boldsymbol{z}+r f_{T}^{2}\right) d t
$$

Where $Q$ and $r$ are the weight matrix and constant for the state and the control force, respectively. The optimal control force in this full state feedback LQR approach is defined by the following standard equation:

$$
f_{T}=-{ }_{r}^{1} \boldsymbol{B}^{T} \boldsymbol{P} \boldsymbol{z}
$$

The matrix $\boldsymbol{P}$ in Eq. (12) is the solution of the following algebraic Riccati equation,

$$
\boldsymbol{P A}+\boldsymbol{A}^{\boldsymbol{T}} \boldsymbol{P}-{ }_{r}^{1} \boldsymbol{P} \boldsymbol{B} \boldsymbol{B}^{\boldsymbol{T}} \boldsymbol{P}+\boldsymbol{Q}=0
$$

There are two major issues in the implementation of this LQR approach. The first issue is that the counterweightrail system is defined by a piecewise linear system of equations. Thus, in a strict sense, this LQR approach is not applicable. More will be said later about this issue. The second issue is related to the classical problem of not being able to measure the full state vector needed in Eq. (12). The classical way to resolve this problem is to use an observer with the LQG approach [3] wherein one estimates the state in terms of measurable response quantities. In vibration of mechanical systems, it is easier to measure accelerations at accessible locations. Herein, it is assumed that three accelerations - one at center of mass of the counterweight, one at bottom, and one at the TMD mass - are measured for feedback. Using Eq. (1) the vector $\boldsymbol{y}_{m}$ related to the measured absolute acceleration responses can be expressed as follows:

$$
\boldsymbol{y}_{\boldsymbol{m}}=\boldsymbol{C}_{y} \boldsymbol{z}+\boldsymbol{D}_{y} f_{T}+\boldsymbol{M}_{i}^{-1} \boldsymbol{f}_{i}+\boldsymbol{v}
$$

where

$$
\boldsymbol{C}_{y}=\left[\begin{array}{ll}
-\boldsymbol{M}_{i}^{-1} \boldsymbol{K}_{i} & -\boldsymbol{M}_{i}^{-1} \boldsymbol{C}_{i}
\end{array}\right] ; \quad \boldsymbol{D}_{y}=\boldsymbol{M}_{i}^{-1} \boldsymbol{D}
$$

and $\boldsymbol{v}$ is a vector of measurement noise. This LQG approach assumes that the input and measurement noise are white noise Gaussian process [3]. For the rail-counterweight system, the acceleration inputs are thus assumed to be Gaussian with intensity ratio $\gamma$ with respect to the measurement noise. The state vector is estimated using the 
measured acceleration feedback from

$$
\boldsymbol{z}=\boldsymbol{A} \boldsymbol{z}+\boldsymbol{B} f_{T}+\boldsymbol{G}_{e}\left(\boldsymbol{y}_{m}-\boldsymbol{C}_{y} \grave{z}-\boldsymbol{D}_{y} f_{T}\right)
$$

where the estimator gain matrix $\boldsymbol{G}_{e}$ is obtained from

$$
\boldsymbol{G}_{e}=\boldsymbol{S}_{e} \boldsymbol{C}_{y}^{T}
$$

in which $\boldsymbol{S}_{e}$ is the solution of the following algebraic Riccati equation

$$
\boldsymbol{A} \boldsymbol{S}_{e}+\boldsymbol{S}_{e} \boldsymbol{A}^{T}-\boldsymbol{S}_{e} \boldsymbol{C}_{y}^{T} \boldsymbol{C}_{y} \boldsymbol{S}_{e}+\gamma \boldsymbol{E} \boldsymbol{E}^{T}=0
$$

The control force is calculated from the LQR algorithm, Eq. (12), developed earlier by substituting the estimated state for the true state as follows:

$$
f_{T}=-{ }_{r}^{1} \boldsymbol{B}^{T} \boldsymbol{P} \hat{z}
$$

In a strict sense, the LQR and LQG approaches described above are only applicable to linear systems. The railcounterweight system is, however, a piece-wise linear system as the system matrix $\boldsymbol{A}$ keeps changing from one state to another because of closing and opening of the gaps in the system. To still use these approaches, here the system matrix pertaining to the case of "all gaps open" is used for calculating the gain matrices and the control force. The response of rail-counterweight system, however, is still calculated with full system nonlinearities described earlier. The proposed approach, thus, only provides sub-optimal gains and control effectiveness. However, to demonstrate that the proposed approach is still effective in reducing the counterweight-rail response, especially in view of the presence of the nonlinearities in the system, numerical simulation studies are conducted.

\subsection{Numerical results - Acceleration feedback}

In the numerical analysis, the intensity of the measurement noise is assumed to be $4 \%$ of the intensity of the input, so that $\gamma=25$. The original non-contact condition of the system is used for matrix $\boldsymbol{A}$ in determining the gain matrices. The control force is limited to $400 \mathrm{lbs}$., which is slightly less than $10 \%$ of the total weight of the counterweight. The numerical results are obtained for the 18.5-lb guide rails attached to bracket supports at each floor levels with story height of $3.66 \mathrm{~m}$. From the catalogs and plans of a leading elevator company, a typical counterweight with $1950 \mathrm{~kg}$ mass is used. The dimensions of the counterweight's frame is $3.505 \mathrm{~m}$ high, $0.711 \mathrm{~m}$ wide and $0.152 \mathrm{~m}$ deep in which the weights fill up to two-third of the height. Other mechanical details about the equivalent stiffness properties of the system are given in Reference [4]. This counterweight system received the seismic input filtered through a ten-story building in which it was located. The building was modeled as a torsional system with three degrees of freedom at each floor level. The building has a first natural frequency of $5.70 \mathrm{rad} / \mathrm{sec}$. The physical properties of this building are also given in Reference [4].

Several values of weight matrix $Q$ and weight factor $r$ were used to obtain the best result. The results presented here are obtained using $\boldsymbol{Q}_{11}=\boldsymbol{Q}_{22}=\boldsymbol{Q}_{33}=\boldsymbol{Q}_{44}=1$ and zero elsewhere and $r=5 \times 10^{-6}$.

To select the tuning frequency for the TMD, we first carried out a numerical study where we calculated the maximum stress in the rails for various frequency values and examined the frequency range that gave the best performance. The frequency ratio is the ratio of the TMD frequency to the fundamental frequency of the counterweight rail system without TMD when all gaps at roller guides are closed and the top roller guides are located at middle of the top story. It is noted that the frequencies of the system change with the position of counterweight along the guide rail and more importantly with the contacts between the restraining plate and the guide rail and contact between the frame and the rail. For instance, the fundamental frequency of the counterweight, tuned mass damper, and rail system in the in-plane motion when the upper roller guides are located at the top of the $10^{\text {th }}$ story building is $11.86 \mathrm{rad} / \mathrm{sec}$ when all gaps are open and it increases to $32.29 \mathrm{rad} / \mathrm{sec}$ when all gaps at the roller guides are closed. If the top roller guides are in the middle of the $10^{\text {th }}$ story, then corresponding frequencies are $11.26 \mathrm{rad} / \mathrm{sec}$ and $28.72 \mathrm{rad} / \mathrm{sec}$, respectively. For this latter case, there is also possibility of contact between the frame and the rail which will increase the frequency to $31.20 \mathrm{rad} / \mathrm{sec}$.

In Fig. 3 we plot the stress ratio as a function of the frequency ratio of the protective systems for two mass ratios. The stress ratio is the ratio of the maximum stresses in the rails with protective system to those without protective 

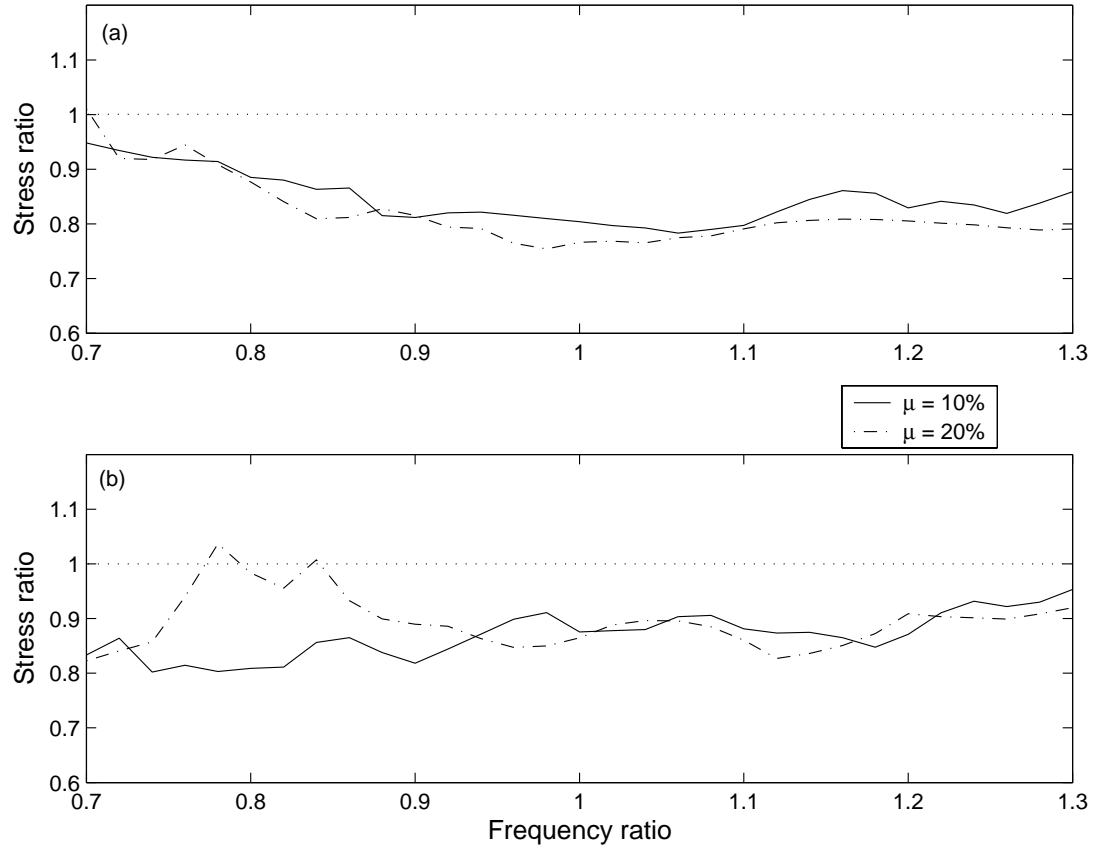

Fig. 3. Ratio of maximum stress as a function of frequency ratio for (a) Northridge earthquake, and (b) El Centro earthquake.

system. The lower stress ratio values in the plots imply better control effectiveness. Figure 3(a) is for the Northridge earthquake motion with maximum ground acceleration of $0.843 \mathrm{~g}$. Figure 3(b) is for the El Centro earthquake motion with maximum ground acceleration of $0.348 \mathrm{~g}$. There is response reduction, but no predictable trend as a function of the frequency ratio is observed. We observe that under Northridge earthquake, the active method does reduce the maximum stress by as much as $25 \%$, especially when TMD mass ratio is $20 \%$ and the frequency ratio is 0.9 or higher. In Fig. 3(b), a similar trend is observed for the El Centro earthquake, but the response reduction is somewhat smaller. In Fig. 4 we show the response reduction obtained for an ensemble of 50 synthetic ground motions. For these results, the TMD with a frequency ratio of 0.9 was chosen. The plotted values are the mean-plus-one-standard-deviation values of the maximum stress in the rails obtained for the ensemble. Here a larger reduction at the higher input intensities is noted. In this figure, we also show the results obtained for the passive TMD - the same TMD but without any actuator. The stress reduction in this case occurs mainly due to the tuned mass damper action. The better performance of the active system compared to the passive system especially in the higher intensity range is noted. The TMDs with $10 \%$ and $20 \%$ mass ratios perform about the same.

In Fig. 5 we show the effect of using active TMD on the system fragility. The system fragility is defined as the probability of failure of the system, considering all positions the counterweight can take along the building height. For each counterweight position, the probabilities of failure were calculated at seven different critical locations on the rails where the maximum stresses occur. These were the locations under the roller guide assemblies, at the center of the rail span, and at the adjacent supports. For calculating these probabilities, the applied maximum stress and the material strength were assumed to be lognormal variable. The means and standard deviations of the maximum stresses at each critical location were obtained using the ensemble response values. The mean strength was taken to be 1.05 times the nominal yield strength and the coefficient of variation of $10 \%$. The failures at these seven locations were considered correlated so that the maximum probability of failure value at one of the seven locations defined the system failure probability. This probability for one position of the counterweight was convolved with the probability of the counterweight being at that position to obtain the total probability of the system failure. Assuming that the elevator can be at any height along the rails with equal probability, the total system probability of failure or the system fragility was the average of the probabilities calculated for different counterweight positions. The fragility results are shown separately in two different part of the figure to get better resolution in the plot for each case. In the middle intensity range, the fragility is seen to have been improved by several orders of magnitude and by about two 


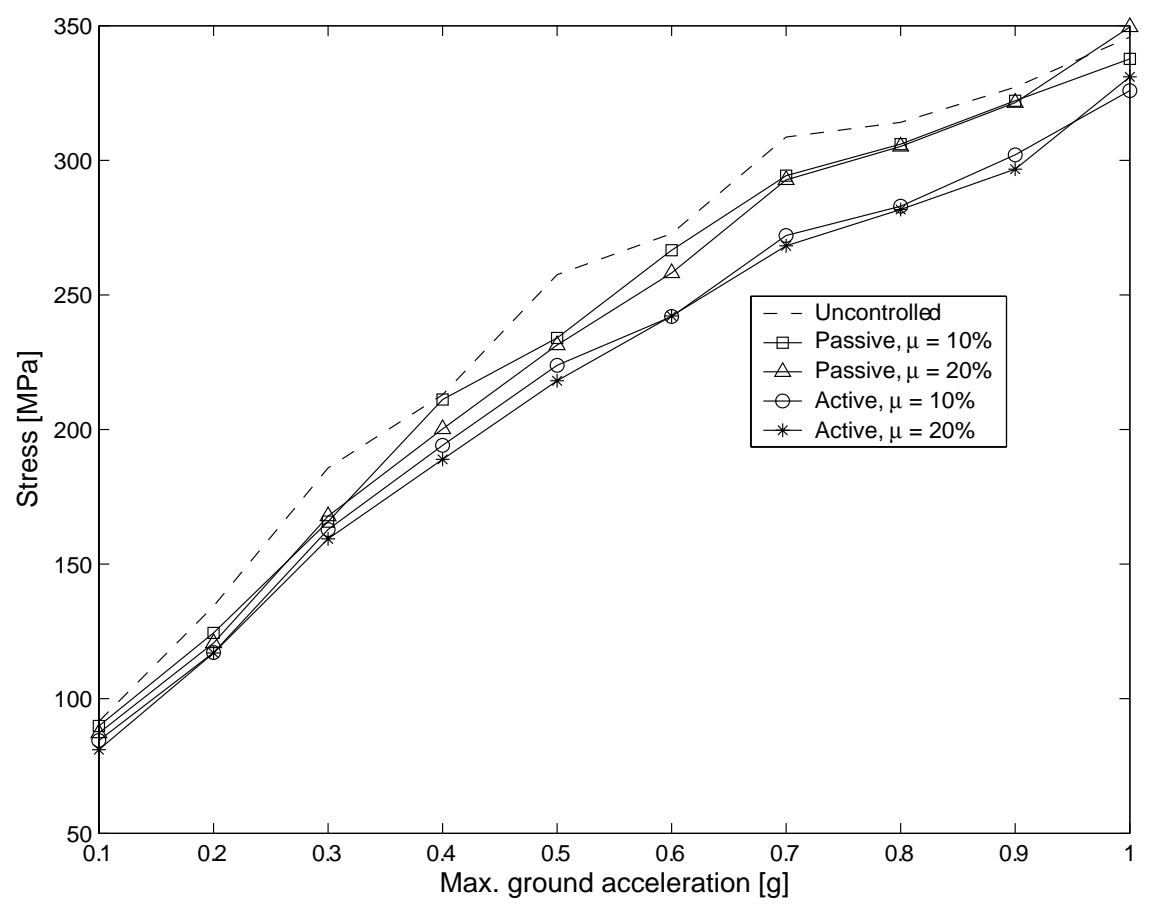

Fig. 4. Mean-plus-one-standard-deviation of the maximum stress in the rail with passive and active devices for an ensemble of synthetic ground motions.

orders of magnitude on the higher end. (The small probability values shown in these figures should not be taken to represent the true system reliabilities as they are functions of the assumed distributions. Rather, they should only be viewed as some measures of the relative effectiveness of the protective system in reducing the fragility.) In general, the active control is seen to improve the performance of the elevator system, and the $20 \%$ ratio mass damper seems perform better than the smaller mass ratio damper.

\section{Semi-active control}

One major disadvantage of using an active system is that it requires an out outside source of power to apply the control force. It may not be a special problem for a small actuator considered in this application. However, to avoid depending upon an external source of power which may not be available during a strong earthquake, here we examine the effectiveness of a semi-active system for controlling a counterweight response. In semi-active control devices one regulates the device properties such as damping level and/or the reactive force. The semi-active devices thus combine the benefits of a passive control device with the ability to actively adapt to the changing response of the structure. The power requirement of semi-active devices is usually much lower than that of the active control devices. They can be easily operated by a small battery pack. As semi-active devices do not input large energy into the system, they cannot destabilize a stable system. The magneto-rheological (MR) dampers are excellent candidates to be as semi-active devices. The MR damper consists of a main cylinder, with a piston and an accumulator, filled with MR fluid with suspended magnetic particles. The magnetic fluid when charged changes its viscosity drastically and almost instantaneously. The MR dampers also provide a stable hysteretic behavior over a broad temperature range [2, 9]. They have, thus, been of special attention in the automotive as well as in the civil engineering applications. In this study we propose to use an MR damper as a semi-active device, and examine its effectiveness to control a counterweight response.

Spencer et al. [9] have proposed a simple mechanical model for the MR damper for analytical investigations. This model, shown in Fig. 6, has been shown to predict the behavior of the MR damper accurately. For this model, the 


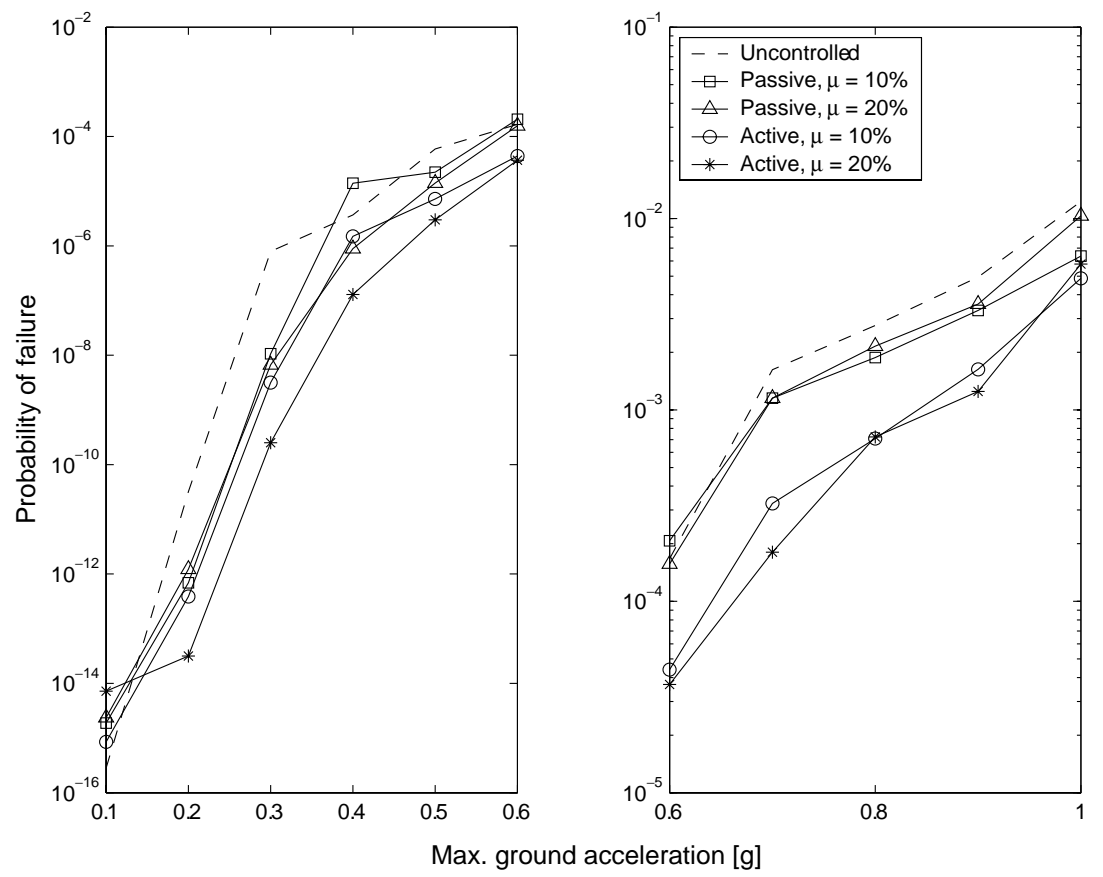

Fig. 5. Fragility curves of the rail for passive and active control schemes.

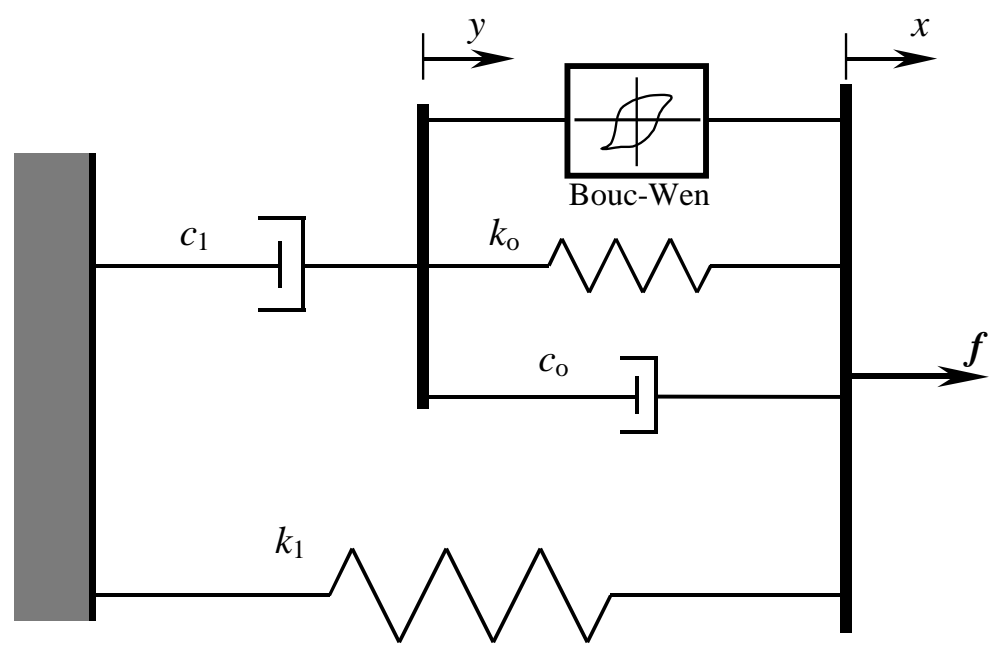

Fig. 6. Mechanical model of a MR damper as proposed by Spencer et al. [9].

force in the damper can be defined in terms of the equations

$$
f=\alpha z+c_{o}(\dot{x}-\dot{y})+k_{o}(x-y)+k_{1}\left(x-x_{o}\right)
$$

This force can also be defined in terms of damper and spring coefficients $c_{1}$ and $k_{1}$ shown in Fig. 6 as:

$$
f=c_{1} \dot{y}+k_{1}\left(x-x_{0}\right)
$$

where $c_{o}, c_{1}, k_{o}$, and $k_{1}$ are the coefficient of the damper and spring shown in Fig. 6. The displacement coordinates $x$ and $y$ are the degrees-of-freedom of the damper, also shown in the same figure. The variable $z$ is the auxiliary variable of the Bouc-Wen element, and $\alpha$ is the coefficient associated with $z$. When attached to the mass damper 
Table 1

Maximum stresses in the rails for various response reduction schemes. TMD frequency ratio $=0.9$

Uncontrolled

$\mu=10 \%$

Passive TMD

Active TMD

MR Damper, passive-on

MR Damper, semi-active

$\mu=20 \%$

Passive TMD

Active TMD

MR Damper, passive-on

MR Damper, semi-active

$$
\begin{array}{cc}
\multicolumn{2}{c}{\text { El Centro (0.348 g) }} \\
\text { Max. stress (MPa) } & \text { Reduction (\%) } \\
199.62 & -
\end{array}
$$$$
177.43
$$$$
163.37
$$$$
165.24
$$$$
150.74
$$$$
177.21
$$$$
169.38
$$$$
153.57
$$

148.25

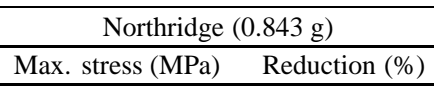

321.25

$308.54 \quad 18.81$

$317.78 \quad 16.37$

$313.30 \quad 17.55$

$309.71 \quad 18.50$

$300.77 \quad 20.85$

$311.41 \quad 18.05$

$\begin{array}{ll}301.69 & 20.61\end{array}$

of the rail-counterweight system, $x$ represents the displacement of the mass damper relative to the frame of the counterweight.

The auxiliary variable $z$ of the Bouc-Wen element is governed by the following equation [11]:

$$
\dot{z}=-\gamma|\dot{x}-\dot{y}| z|z|^{n-1}-\beta(\dot{x}-\dot{y})|z|^{n}+A(\dot{x}-\dot{y})
$$

where $\gamma, \beta, A$, and $n$ are the Bouc-Wen model parameters which control the shape of the hysteresis loops of the damper element.

Using Eqs (20) and (21), we obtain the following equation for the degree-of-freedom $y$ :

$$
\dot{y}=c_{c_{o}+c_{1}}^{1}\left[\alpha z+c_{o} \dot{x}+k_{o}(x-y)\right]
$$

Spencer et al. [9] suggested the dependence of the force on the applied voltage through the linear relationship between the dashpots and the Bouc-Wen element in the mechanical model and the applied voltage as:

$$
\begin{aligned}
& \alpha=\alpha_{a}+\alpha_{b} u \\
& c_{o}=c_{o a}+c_{o b} u \\
& c_{1}=c_{1 a}+c_{1 b} u
\end{aligned}
$$

where $u$ is the output of the first order filter for the commanded voltage $v$ :

$$
\dot{u}=-\eta(u-v)
$$

To solve the equations of motion of the rail-counterweight system with mass damper and MR damper device, Eqs (22), (23) and (27) are added to the original equations of motion, where the control force $f_{T}$ is now replaced by the force from the MR damper shown in Eq. (20) or Eq. (21). The complete set of equations needed for the semi-active control are summarized below:

$$
\begin{aligned}
& \dot{\boldsymbol{z}}=\boldsymbol{A} \boldsymbol{z}+\boldsymbol{B} f_{T}+\boldsymbol{E} \ddot{y}_{i}+\boldsymbol{f}^{*} \\
& f_{T}=c_{1} \dot{y}+k_{1}\left(x-x_{o}\right) \\
& \dot{z}=-\gamma|\dot{x}-\dot{y}| z|z|^{n-1}-\beta(\dot{x}-\dot{y})|z|^{n}+A(\dot{x}-\dot{y}) \\
& \dot{y}=c_{o}+c_{1}\left[\alpha z+c_{o} \dot{x}+k_{o}(x-y)\right] \\
& \dot{u}=-\eta(u-v)
\end{aligned}
$$

For a given voltage one can calculate the response of the system using these equations. The objective is, however, to choose the appropriate voltage to reduce the response. For this, we used the clipped-optimal control algorithm, 


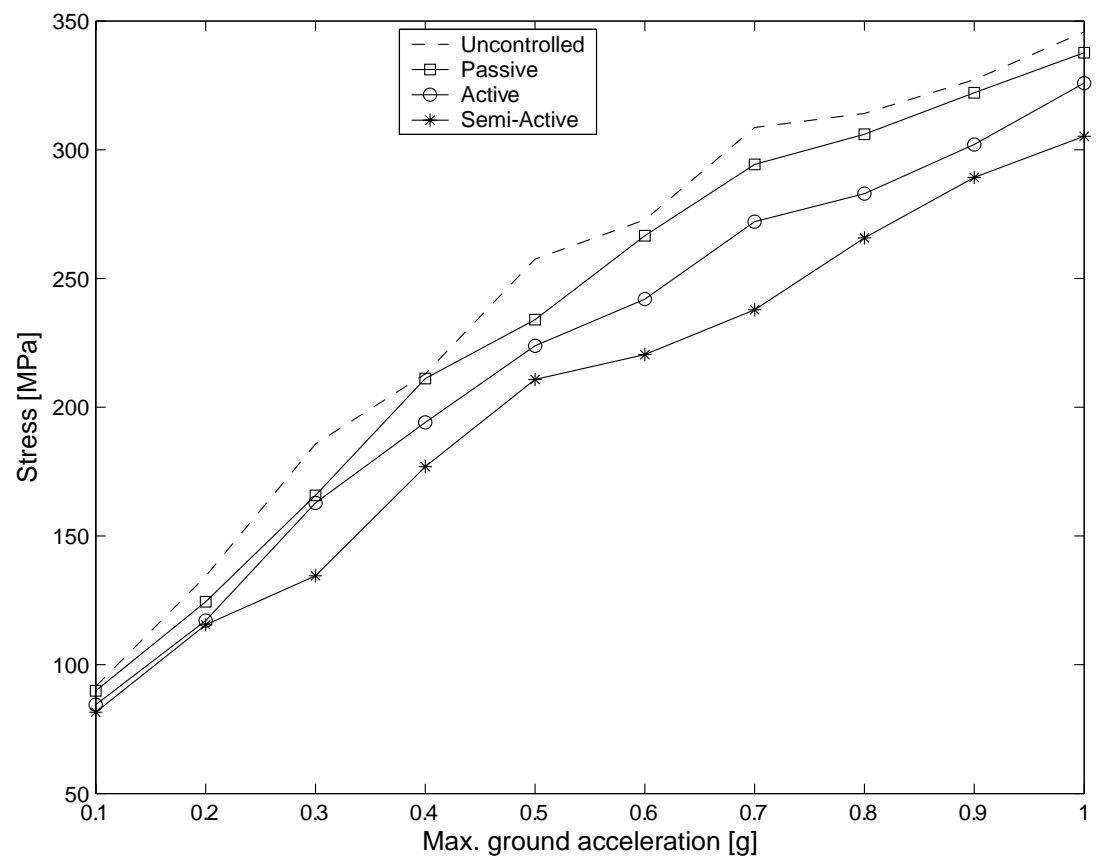

Fig. 7. Mean-plus-one-standard-deviation of the maximum stress in the rails for passive, active and semi-active devices with $10 \%$ mass ratio damper for an ensemble of synthetic ground motions.

also used by Dyke et al. [2]. In this control scheme, the required optimal control force is calculated by the LQG algorithm described earlier and compared with the force provided by the MR damper. If the required force is more than the damper force and is in the same direction, then maximum voltage is applied. If the require force is equal to the present damper force, then also the maximum voltage is applied. On the other hand if the required optimal force is less or in opposite direction, then the voltage is turned off. The effectiveness of this control scheme is now examined numerically on the same counterweight-rail system considered earlier.

\subsection{Numerical results - Semi-active control}

The following numerical results are obtained for a prototype MR damper with $3000 \mathrm{~N}$ (674 lbs) maximum force capacity. The parameters needed to characterize the force deformation characteristics of this damper are defined by Dyke et al. [2]. The force range and the size of the device $(3.8 \mathrm{~cm}$ diameter, $21.5 \mathrm{~cm}$ extended length, and $\pm 2.5 \mathrm{~cm}$ stroke) make it quite suitable for use on a rail-counterweight system. The maximum voltage for this MR damper is $2.25 \mathrm{~V}$ as the saturation effect begins at voltage higher than $2.25 \mathrm{~V}$ [2].

In Table 1 we present the maximum stress in the rails using different response control methods including the semi-active approach described above. The upper part of the table is for a $10 \%$ mass ratio damper and the lower part is for the larger $20 \%$ mass ratio damper. The results for two well-known as recorded earthquake induced ground motions - Northridge and El Centro earthquakes - are presented. The columns showing the percent reduction in the maximum stress response caused by the control approaches are provided. The results shown are for different control options: (a) Uncontrolled; (b) Passive TMD where no actuation device is used; (c) Active TMD (the results are the same as those presented in the first part of this paper); (d) MR Damper-Passive On when the damper with full voltage is used and; (e) MR Damper - Semi-active where the voltage to the MR damper is turned on and off according to the clipped-optimal control described in the previous section. The comparison of these results shows that the semi-active actuation does seem to provide somewhat better response reduction compared to other approaches especially for the El Centro earthquake motion. These variations in the control effectiveness for individual earthquake motions are expected. In the next set of results we now consider the ensemble of 50 earthquake accelerograms, also used earlier. Figure 7 shows the mean-plus-one-standard-deviation of the maximum stress obtained under different control 


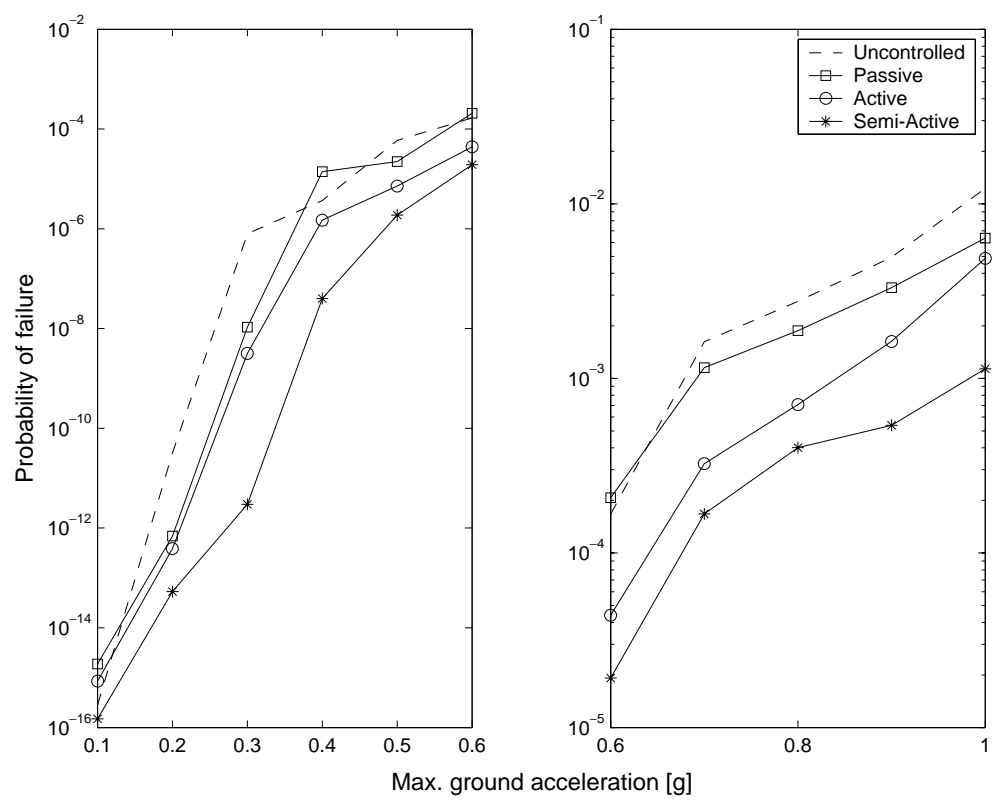

Fig. 8. Fragility curves of the rail for different control methods.

scenarios using a $10 \%$ mass ratio damper. Here we clearly note that all active schemes reduce the stresses over the range of excitation intensities, and the semi-active scheme performs the best of the three approaches considered. The effect of this response reduction on the system fragility is shown in Fig. 8. Here again we present these results separately for the lower and higher intensity ranges. In the lower intensity ranges the fragility is improve by several orders of magnitude around the intensities of 0.3 to $0.4 \mathrm{~g}$ values, where as the fragility improvements in the higher intensity ranges are of an order of magnitude. Please also note the comment made earlier about the low probability values in Fig. 5. In Fig. 9 we also show the plot of the absolute maximum and average of the maximum displacements of the TMD mass relative to the frame obtained for an ensemble of ground motions considered in the study as a function of the intensity of ground motion. This displacement is also the stroke of the actuator plunger. The figure shows these displacements for both the acceleration feedback as well as semi-active case. At the highest intensity of motion considered in this study, this maximum value of the plunger stroke is about $8 \%$ of the counterweight width. This is higher than the stroke specification of the MR damper used in the numerical study, but does not seem to be too large for an actuator or the MR damper design that can be accommodated in the counterweight frame.

\section{Concluding remarks}

Two control schemes for use with the counterweight-rail systems have been considered in this study: (1) a fully active scheme using acceleration-feedback with LQG algorithm and (2) a semi-active scheme with MR dampers that combines the benefit of passive and active dampers. Numerical results are presented to demonstrate their effectiveness in a seismic environment. It is shown that these methods can reduce the seismic response and improve the performance of rail-counterweight systems.

The numerical results show that the semi-active approach is better than, or at least as effective as, the acceleration feedback approach. This approach has a special advantage over the fully active approach that it does not need an outside source of power; a semi-active system using an MR damper can always be activated by a simple battery pack. It is also felt that the performance of the semi-active scheme could be further improved by using a more refined algorithm to regulate the MR damper than a simple on-off control scheme used in this study. The possibility of doing this, however, was not examined further in this study. 


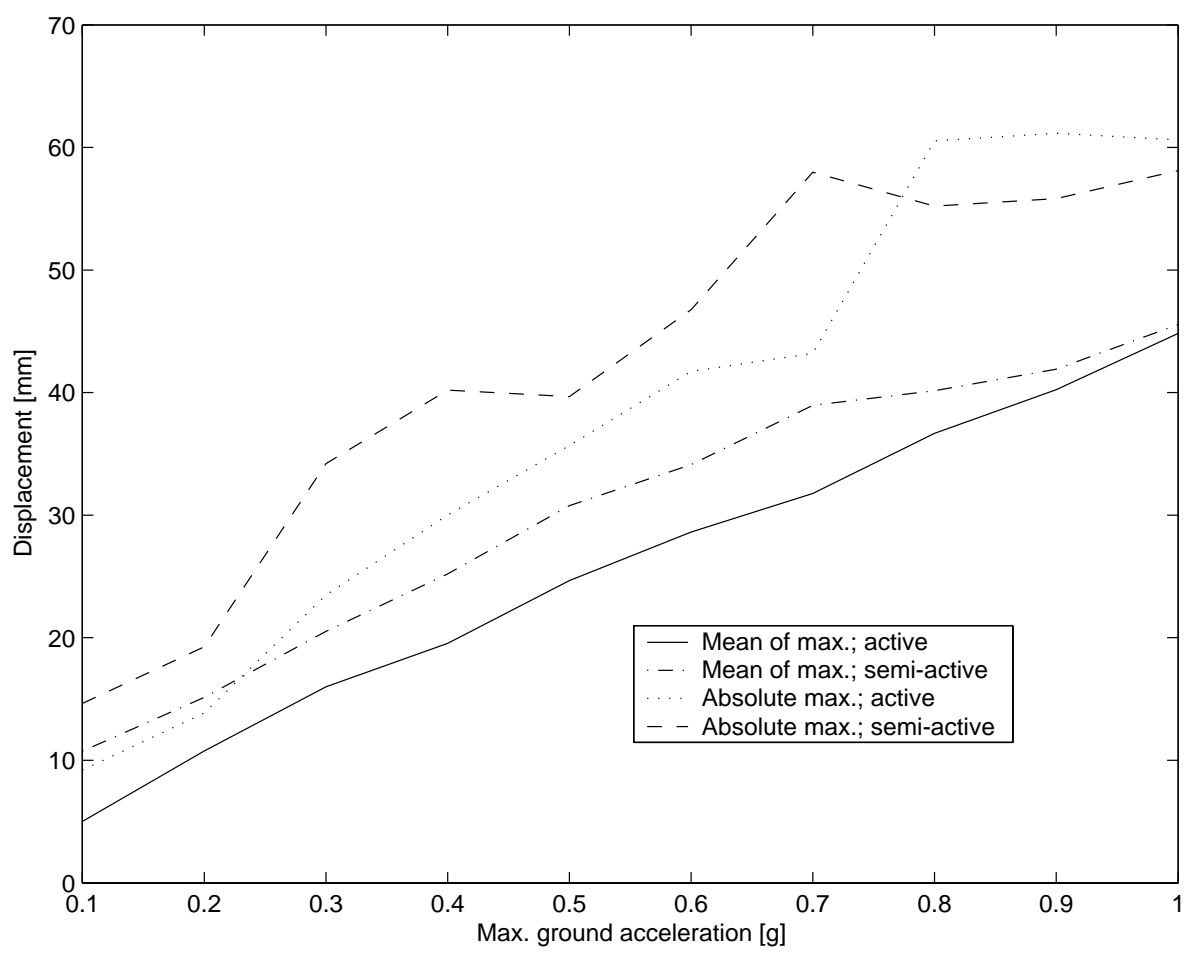

Fig. 9. The absolute maximum and average of the maximum actuator stroke values calculated for the ensemble of time histories for different levels of ground motion intensities.

\section{Acknowledgment}

This research is supported by National Science Foundation through grant no. CMS-0201475. This support is gratefully acknowledged. Any opinion, findings, and conclusions or recommendations expressed in this study are those of the writers and do not necessarily reflect the views of the National Science Foundation.

\section{References}

[1] American Society of Mechanical Engineers, Safety code for elevators and escalators ASME A 17.1, ASME, New York, 1996.

[2] S.J. Dyke, B.F. Spencer Jr., M.K. Sain and J.D. Carlson, Modeling and control of magnetorheological dampers for seismic response reduction, Smart Materials and Structures 5 (1996), 565-575.

[3] H. Kwakernaak and R. Sivan, Linear Optimal Control Systems, John Wiley \& Sons, New York, 1972.

[4] Rildova, Seismic performance of rail-counterweight system of elevator in buildings, Ph.D. Dissertation, Virginia Polytechnic Institute and State University, 2004.

[5] A.J. Schiff, H.S. Tzou and Y.H. Chu, Earthquake response of elevator counterweights, Proc. th World Conference on Earthquake Engineering 8 (1980), 483-486.

[6] M.P. Singh, L.E. Suarez and Rildova, Seismic response of rail-counterweight systems in elevators, Earthquake Engineering and Structural Dynamics 31 (2002), 281-303.

[7] M.P. Singh, Rildova and L.E. Suarez, Non-linear seismic response of the rail counterweight system in elevators in buildings, Earthquake Engineering and Structural Dynamics 33 (2004), 249-270.

[8] B.F. Spencer Jr., S.J. Dyke, M.K. Sain and J.D. Carlson, Phenomenological model for magnetorheological dampers, Journal of Engineering Mechanics 123 (1997), 230-238.

[9] H.S. Tzou and A.J. Schiff, Structural dynamics of elevator counterweight system and evaluation of passive constraints, Journal of Structural Engineering 114 (1988), 783-803.

[10] H.S. Tzou and A.J. Schiff, Dynamics and control of elevators with large gaps and rubber dampers, Journal of Structural Engineering 115 (1989), 2753-2771.

[11] Y.K. Wen, Method of random vibration of hysteretic systems, Journal of Engineering Mechanics Division 102 (1976), 249-263. 
[12] T.Y. Yang, H. Kullegowda, R.K. Kapania and A.J. Schiff, Dynamic response analysis of elevator model, Journal of Structural Engineering 109 (1983), 1194-1210. 

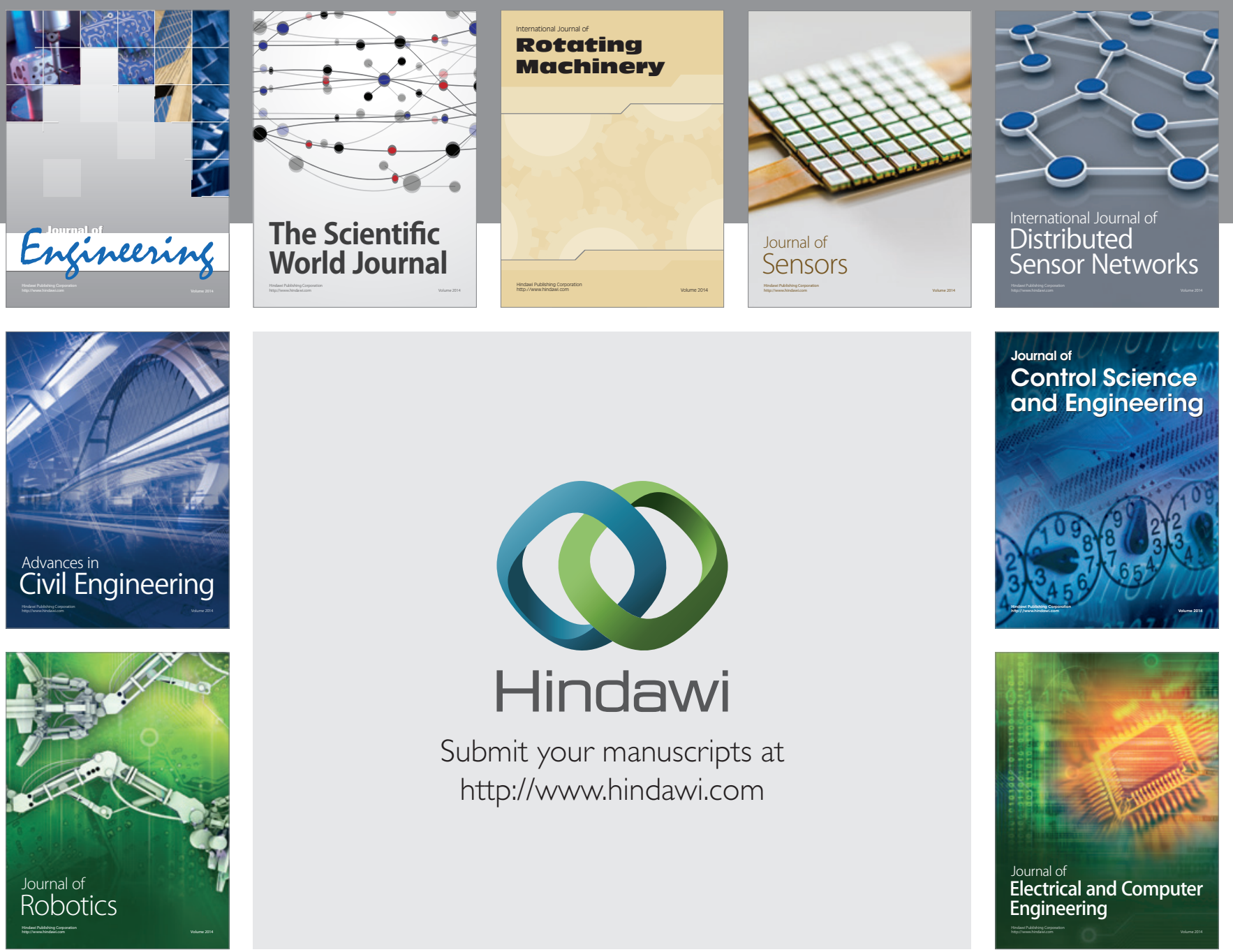

Submit your manuscripts at

http://www.hindawi.com
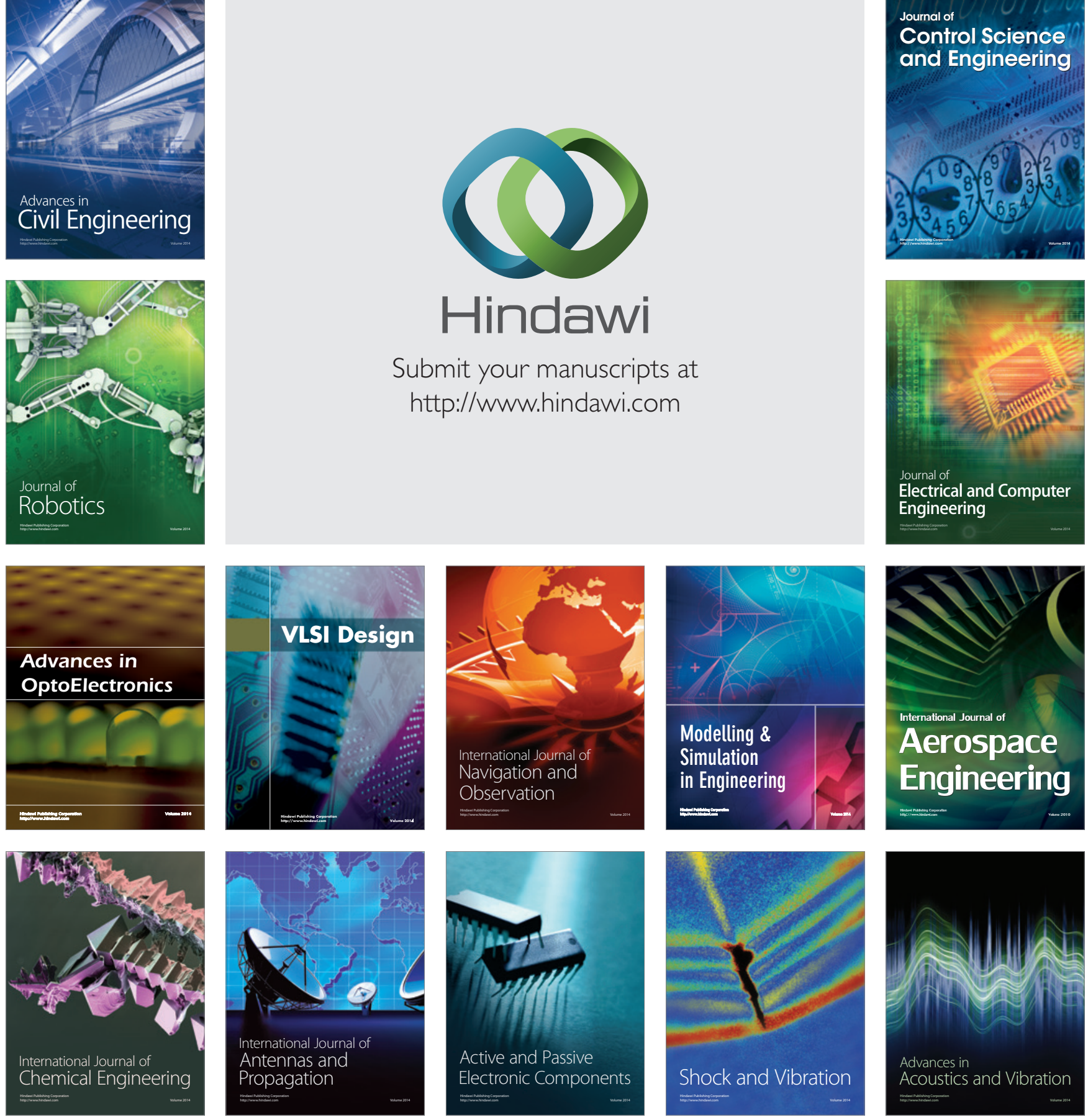